\title{
Phronesis
}

\section{Les reliances de l'alternance en formation et ses effets sur les processus de construction identitaire des alternants}

\section{Philippe Maubant et Pascal Roquet}

Volume 5, numéro 1, 2016

Les reliances de l'alternance en formation et ses effets sur les processus de construction identitaire des alternants

URI : https://id.erudit.org/iderudit/1037189ar

DOI : https://doi.org/10.7202/1037189ar

Aller au sommaire du numéro

Éditeur(s)

Université de Sherbrooke

ISSN

1925-4873 (numérique)

Découvrir la revue

Citer ce document

Maubant, P. \& Roquet, P. (2016). Les reliances de l'alternance en formation et ses effets sur les processus de construction identitaire des alternants.

Phronesis, 5(1), 1-3. https://doi.org/10.7202/1037189ar d'utilisation que vous pouvez consulter en ligne. 


\section{INTRODUCTION}

Philippe MAUBANT* , , Pascal ROQUET**

*Université de Sherbrooke 2500 boul. de l'Université

Sherbrooke, Canada

J1K 2R1

Philippe.Maubant@USherbrooke.ca
*Conservatoir national des arts et métiers 292 Rue Saint-Martin, 75003 Paris, France

pascal.roquet@cnam.fr

\section{Les reliances de l'alternance en formation et ses effets sur les processus de construction identitaire des alternants}


L'alternance, figure éducative et pédagogique des politiques et des dispositifs de formation professionnelle, est aujourd'hui, à nouveau, omniprésente dans les discours politiques et sociaux visant à défendre un rapprochement plus significatif et plus efficace entre la formation et l'emploi. Se redéployant sous l'argumentaire des injonctions à la professionnalisation, l'alternance constitue une dimension structurante de la formation professionnelle. Elle poursuit son mandat de réconcilier l'école et la vie (Houssaye, 1987). Elle est marquée de toutes les espérances dans sa capacité à rapprocher, voire à mieux articuler les situations de formation et les situations de travail. Elle porte aussi en elle d'autres ambitions en interpelant différentes problématiques, comme celle de la pédagogie par alternance (Maubant, 2007), de la question des temporalités en formation (Roquet et al., 2013) ou bien encore celle des pratiques formatives de certains acteurs de l'alternance (Correa Molina et Gervais, 2008; Mazalon, Gagnon et Roy, 2014), comme les superviseurs ou les tuteurs.

L'alternance constitue aussi un slogan et une figure éducative. En effet, elle participe des discours sociaux sur et pour la professionnalisation (Demazière, Roquet, Wittorski, 2012). À cet égard, elle contribue à une réorganisation des curricula et des dispositifs de formation dans et par les situations de travail. Le slogan de l'alternance semble donc intimement lié au discours politique et organisationnel sur la professionnalisation. Mais elle est aussi une figure éducative de la professionnalisation dans la mesure où elle est porteuse d'une ambition pédagogique, celle de favoriser et de faire réussir les apprentissages des « alternants ». La valeur ajoutée attendue de l'alternance est donc tout autant politique et stratégique qu'éducative et pédagogique. L'alternance est aujourd'hui engagée dans sa propre institutionnalisation. Elle se définit dans le cadre de réponses globales aux problématiques d'insertion et de formation tout au long de la vie et se caractérise par une grande hétérogénéité de formes et de pratiques. L'institutionnalisation de l'alternance est aussi une question de construction de sens : sens identitaire, sens social, sens éducatif et sens pédagogique

Il demeure que pour penser et mettre en œuvre l'alternance en formation un construit et un paradigme semblent constamment présents, en filigrane, lorsque l'on étudie les discours sur l'alternance : le construit de reliance (Morin, 1977, Le Moigne, 2008) et le paradigme de la coopération. La reliance semble en effet un construit représentatif et symbolique du paradigme de la coopération structurant les rapports entre les différents acteurs de l'alternance. En effet, pour penser et mettre en œuvre l'alternance en formation, une condition conceptuelle et paradigmatique semble devoir être présente.

Certes, il s'agit de favoriser la mise en relation, l'émergence d'interrelations, la stimulation d'interactions entre les différents acteurs de la formation, et implicitement, entre les différentes situations potentiellement formatives, entre les différents espaces et temps constitutifs du processus de professionnalisation. Mais il s'agit aussi de donner sens à ces reliances à partir de figures ou de modèles comme celui de la coopération par exemple. Soutenir le recours au construit de reliance et au paradigme de la coopération contribue sans doute à envisager les reliances sous la forme d'un dialogue, voire d'une tension dialectique entre protagonistes et entre situations, tension qui interroge de fait le sens et la forme même de la coopération dans un contexte idéologique, politique et socio-organisationnel faits d'injonctions à la coopération, mais aussi à la collaboration et au partenariat. Définir, décrire et caractériser les reliances conduit donc à débattre du statut, du sens et de la forme de ces concepts et construits, en considérant par exemple qu'un mode de collaboration entre acteurs de l'alternance peut être de type coopératif. Dès lors, définir cette collaboration de type coopératif constitue selon nous un cadre pertinent pour convoquer le construit de reliance, instruire son procès au sens de débattre de son usage et de sa pertinence. De fait, analyser le construit de reliance dans et par une mise en question du statut, du sens et des formes de la coopération nous permet de redessiner la figure éducative et péda- 
gogique de l'alternance en formation, tant sur le plan de sa construction macro-politique et macro-stratégique que sur le plan méso-organisationnel. C'est le sens de ce numéro de Phronesis.

En proposant un débat croisé sur le construit de reliance et sur le paradigme de la coopération, nous cherchons in fine à comprendre les effets de ces reliances sur les processus de constructions et de transformation identitaires des acteurs de l'alternance. Ainsi ce numéro propose-t-il à répondre aux questions suivantes :

1. Quels impacts le recours au construit de reliance a-t-il sur la manière de penser, de décrire, de caractériser et de comprendre la coopération dans et par les formations par alternance?

2. De quelles manières et à quelles conditions l'usage du construit de reliance permet-il de saisir les processus de construction et de transformation identitaires des acteurs de l'alternance?

3. Le recours au construit de reliance permet-il de penser autrement l'alternance en formation ?

4. L'usage du construit de reliance pour décrire et comprendre les différentes configurations de l'alternance nécessite-il de préciser préalablement la coopération dans sa forme organisationnelle mais aussi dans sa forme pédagogique?

\section{Références bibliographiques:}

Correa Molina, E., Gervais, C. (2008). Les stages en formation à l'enseignement : pratiques et perspectives théoriques. Québec : Presses de l'Université du Québec.

Demazière, D., Roquet, P. \& Wittorski, R. (2012). (dir.). La professionnalisation mise en objet. Paris : L'Harmattan.

Houssaye, J. (1987). École et vie active. Résister ou s'adapter? Neufchâtel : Delachaux et Niestlé.

Le Moigne, J.-L. (2008). Edgar Morin. Le génie de la reliance. Synergies-Monde, (4), 177-184

Maubant, P. (2007). Penser l'alternance comme logique de professionnalisation des enseignants. Dans Vanhulle, S., Merhan, F. \& Ronveaux, C. Alternances en formation. Raisons éducatives (11), 67-82.

Mazalon, É. Gagnon, C., Roy, S. (2014). L'encadrement des stagiaires en milieu de travail : étude exploratoire dans un cadre formel d'alternance en formation professionnelle initiale. Éducation et francophonie, XLII (1),113-135.

Morin, É. (1977). La méthode. T1. Paris : Le Seuil.

Roquet, P., Goncalves, M.J., Roger, L., Viana-Caetano, A.P. (2013). Temps, temporalités et complexité dans les activités éducatives et formatives. Paris : L'Harmattan 